\title{
Effect of vasopressin antagonism on renal handling of sodium and water and central and brachial blood pressure during inhibition of the nitric oxide system in healthy subjects
}

\author{
Safa Al Therwani", Frank Holden Mose, Janni Majgaard Jensen, Jesper Nørgaard Bech \\ and Erling Bjerregaard Pedersen
}

\begin{abstract}
Background: Tolvaptan is a selective vasopressin receptor antagonist (V2R) that increases free water excretion. We wanted to test the hypotheses that tolvaptan changes both renal handling of water and sodium and systemic hemodynamics during basal conditions and during nitric oxide (NO)-inhibition with L-NG-monomethyl-arginine (L-NMMA).

Methods: Nineteen healthy subjects were enrolled in a randomized, placebo-controlled, double-blind, crossover study of two examination days. Tolvaptan $15 \mathrm{mg}$ or placebo was given in the morning. L-NMMA was given as a bolus followed by continuous infusion during 60 minutes. We measured urine output(UO), free water clearance $\left(\mathrm{C}_{\mathrm{H} 2 \mathrm{O}}\right)$, fractional excretion of sodium ( $\left(\mathrm{EE}_{\mathrm{Na}}\right)$, urinary aquaporin-2 channels (u-AQP2) and epithelial sodium channels $\left(u-E N a C_{\gamma}\right)$, plasma vasopressin ( $\left.p-A V P\right)$, central and brachial blood pressure(cBP, bBP).

Results: During baseline conditions, tolvaptan caused a significant increase in $\mathrm{UO}, \mathrm{C}_{\mathrm{H} 2 \mathrm{O}}$ and $\mathrm{p}-\mathrm{AVP}$, and $\mathrm{FE}_{\mathrm{Na}}$ was unchanged. During L-NMMA infusion, $\mathrm{UO}$ and $\mathrm{C}_{\mathrm{H} 2 \mathrm{O}}$ decreased more pronounced after tolvaptan than after placebo ( -54 vs. $-42 \%$ and -34 vs. $-9 \%$ respectively). U-AQP2 decreased during both treatments, whereas u-ENaC $\mathrm{E}_{\gamma}$ decreased after placebo and increased after tolvaptan. CBP and bBP were unchanged.

Conclusion: During baseline conditions, tolvaptan increased renal water excretion. During NO-inhibition, the more pronounced reduction in renal water excretion after tolvaptan indicates that NO promotes water excretion in the principal cells, at least partly, via an AVP-dependent mechanism. The lack of decrease in u-AQP2 by tolvaptan could be explained by a counteracting effect of increased plasma vasopressin. The antagonizing effect of NO-inhibition on u-ENaC suggests that NO interferes with the transport via ENaC by an AVP-dependent mechanism.
\end{abstract}

Keywords: Tolvaptan, Nitric oxide, AQP2, ENaC, Blood pressure, AVP, Renin, Angiotensin II

\section{Background}

Vasopressin (AVP) stimulates translocation of aquaporin2 water channels (AQP2) to the apical plasma membrane, thereby increasing water permeability in the collecting ducts [1-3]. In addition to its effects on water permeability AVP also stimulate sodium absorption in the collecting ducts by increasing the activity of the eprthelial sodium

\footnotetext{
*Correspondence: safatherwani@hotmail.com

University Clinic in Nephrology and Hypertension, Department of Medical Research, Holstebro Hospital and Aarhus University, Hospital Unit Jutland West, Laegaardvej 12, 7500 Holstebro, Denmark
}

channels $(\mathrm{ENaC})[4,5]$. In addition to AVP, recent studies have suggested that nitric oxide (NO) also has an effect on the translocation of AQP2 to form water channels [6-8]. The synthesis of NO is inhibited by L-NG-monomethyl-arginine (L-NMMA), a competitive inhibitor of NO. Infusion of L-NMMA causes reduced renal plasma flow, reduced renal excretion of sodium, renal renin concentration and increased blood pressure [7,9]. However, the effects of NO inhibition on the translocation of AQP2 water channels during inhibition of the vasopressin 2 receptor (V2R) have not been examined. 
Tolvaptan is a selective V2R antagonist that inhibits activation of V2R by AVP thereby inhibiting insertion of AQP2 water channels in the collecting ducts, and thus stimulating aquaresis, an electrolyte-free water excretion [2,10-19]. We wanted to test the hypotheses that short-term tolvaptan treatment would decrease renal absorption of water and sodium during basal conditions, and that this effect would be antagonized by NO-inhibition. In addition, we wanted to test whether the effects of tolvaptan on the central blood pressure, vasopressin in plasma and the activity of the renin-angiotensin-aldosterone system antagonized the effects on renal water and sodium handling.

Selctive V2R antagonism may be therapeutically useful for the treatment of conditions with impaired water excretion, and consequently dilutional hyponatremia, associated with the syndrome of inappropriate secretion of antidiuretic hormone, congestive heart failure and cirrhosis. Increased knowledge of the mechanisms involved in the regulation of water and sodium may improve future treatment of hyponatremia in these patients.

We conducted a randomized, placebo-controlled, doubleblinded, crossover study in healthy subjects to measure the effects of tolvaptan on (1) renal handling of water and sodium (glomerular filtration rate (GFR), urine output (UO), free water clearance $\left(\mathrm{C}_{\mathrm{H} 2 \mathrm{O}}\right)$, u-AQP2, u-EnaC $\gamma$, fractional excretion of sodium $\left(\mathrm{FE}_{\mathrm{Na}}\right)$, (2) plasma concentrations of renin (PRC), angiotensin II (p-AngII), aldosterone (p-Aldo) and p-AVP, (3) hemodynamics (brachial blood pressure (bBP), central blood pressure (cBP), pulse wave velocity (PWV) and augmentation index (AI)), during baseline conditions and during and after inhibition of the nitric oxide synthesis.

\section{Methods}

Subjects

\section{Inclusion criteria}

Healthy subjects were enrolled with the inclusion criteria 1) age between 18-40 yrs., 2) men and women, 3) nonsmokers, 4) BMI between 18.5 and $30 \mathrm{~kg} / \mathrm{m}^{2}$.

\section{Exclusion criteria}

Exclusion criteria included 1) arterial hypertension (bBP > $140 \mathrm{mmHg}$ systolic and/or $>90 \mathrm{mmHg}$ diastolic), 2) history or clinical signs of neoplastic disease or disease of the heart, lungs, kidneys or endocrine organs, 3) drug or alcohol abuse (I.e. $>14$ objects a week for women and 21 for men), 4) medical treatment except oral contraceptives, 5) pregnancy or breast-feeding, 6) significant laboratory abnormalities in the screening test of blood samples (I .e. abnormal haemoglobin, white cell count, plasma sodium, plasma potassium, plasma creatinine, plasma albumin, plasma bilirubin, plasma alanine aminotransferase or serum cholesterol) and urine samples (I.e. albuminuria or glucosuria), 7) abnormal electrocardiogram, 8) blood donation less than one month prior to the study.
In fertile women contraceptive treatment must be used during and after the after the study period (I.e. p-pills, spiral, depot injection of gestagen, sub dermal implantation, hormonal contraceptive vaginal ring and transdermal contraceptive patch).

Withdrawal criteria were development of one or more of the exclusion criteria, bBP increase above 180/105 $\mathrm{mmHg}$ during infusion of L-NMMA, withdrawal of consent or lack of compliance.

\section{Study design}

We performed a randomized, crossover double-blinded, placebo- controlled trial. The trial consisted of two treatment periods, placebo or tolvaptan, with an intermediate wash- out period of at least two weeks to eliminate any carryover effects.

\section{Number of subjects}

$\mathrm{C}_{\mathrm{H} 2 \mathrm{O}}$ was used as the main effect variable. With a minimal relevant difference of $6 \mathrm{ml} / \mathrm{min}$ with an estimated standard deviation (SD) of $4 \mathrm{ml} / \mathrm{min} 18$ subjects were needed using a level of significance of $5 \%$ and a statistical power of $80 \%$. Due to possible drop outs 20 subjects were included.

\section{Recruitment}

Healthy subjects were recruited by advertising in public institutions and private companies.

\section{Ethics}

This study was approved by the Regional Committee on Health Research Ethics (j. no. M-201223912). The study was conducted in conformity with the principles of the declaration of Helsinki. Written informed consent was obtained from all subjects.

\section{Effect variables}

The primary effect variable was $\mathrm{C}_{\mathrm{H} 2 \mathrm{O}}$. The secondary effect variables were 1) renal function (GFR, UO, u-AQP2, u$\mathrm{ENaC}_{\gamma}, \mathrm{FE}_{\mathrm{Na}}$ ), 2) hemodynamics (bBP, cBP, PWV, AI) and 3) vasoactive hormones (PRC, p-ANG II, p-Aldo, p- AVP).

\section{Diet}

Four days prior to each treatment period all subjects ingested a standardized diet of $11.000 \mathrm{KJ}$. The diet was composed of $55 \%$ carbohydrates, $15 \%$ proteins and $30 \%$ fat following general dietary guidelines. The sodium content was $150 \mathrm{mmol}$ per day. No additional sodium or other spices was allowed. The daily fluid intake was 2.5 L. No alcohol intake was allowed.

\section{Experimental procedure}

The examinations were conducted in Medical Research from 7:45 AM to 1:00 PM. The procedures were identical on the two examination days. On the morning of each 
examination day, subjects were given placebo or tolvaptan $15 \mathrm{mg}$ at 6.00 AM. Prior to each examination day a 24hours urine was collected and a fasting period of eight hours was performed.

An intravenous catheter was placed in each arm to collect blood samples and infuse the ${ }^{51} \mathrm{Cr}$-EDTA. At 8:00 AM and every 30 minutes, an oral water load of $175 \mathrm{ml}$ was given.

$\mathrm{BP}$ was measured every 30 minutes from 8:30 AM to 1:00 PM. A bolus infusion of L-NMMA $4.5 \mathrm{mg} / \mathrm{kg}$ was given at 11:00 AM, followed by continuous infusion ( $3 \mathrm{mg} / \mathrm{kg} /$ hour) during one hour. The dose was based on results from a dose-finding study made by our team in healthy subjects [9]. During infusion of L-NMMA BP was measured every 5 minutes, and every 15 minutes after infusion of L-NMMA.

Blood samples were drawn every 30 minutes from 8:30 to 1:00 PM and were analyzed for $\mathrm{p}-\mathrm{Na}, \mathrm{p}-\mathrm{osm}, \mathrm{p}-$ creatinine, p-albumin and $\mathrm{p}-{ }^{51} \mathrm{Cr}$-EDTA. Blood samples were drawn for measurement of PRC, p-Aldo, p-Ang II and p-AVP every 60 minutes; at 11:00 AM (baseline), at 12:00 AM (after end of L-NMMA infusion) and at 1:00 PM (60 minutes after end of L-NMMA infusion).

Urine samples were collected by voiding in standing or sitting position every 30 minutes from 9:30 AM to 1:00 PM after collecting BP measurements and blood samples. Otherwise subjects were kept in a supine position in a quiet, temperature-controlled room $\left(22-25^{\circ} \mathrm{C}\right)$. Baseline period were means of the first three clearance periods. The urine samples were analyzed for osmolality (u-osm), creatinine (u-creatinine), sodium (u-Na), u-AQP2, u-EnaC $C_{\gamma}$ and ${ }^{51} \mathrm{Cr}$-EDTA $\left(\mathrm{u}-{ }^{51} \mathrm{Cr}\right.$-EDTA).

Applanation tonometry with SphygmoCor was performed from 10:40 to 11:40 AM.

\section{Measurements}

\section{Renal function}

GFR were measured using the constant infusion clearance technique with 51Cr-EDTA as reference substance.

$\mathrm{C}_{\mathrm{H} 2 \mathrm{O}}$ was determined using the formula $\mathrm{C}_{\mathrm{H} 2 \mathrm{O}}=\mathrm{UO}-\mathrm{C}_{\mathrm{osm}}$, where $\mathrm{C}_{\mathrm{osm}}$ is the osmolar clearance.

Clearance $(\mathrm{C})$ of substance $\mathrm{X}$ was calculated as $\mathrm{C}_{\mathrm{X}}=$ $\mathrm{U}_{\mathrm{X}} /\left(\mathrm{P}_{\mathrm{X}} \mathrm{x} U \mathrm{UO}\right)$, where $\mathrm{U}_{\mathrm{X}}$ denotes concentration of $\mathrm{x}$ in urine, $P_{X}$ denotes concentration of $x$ in plasma, and $U O$ is urine excretion rate.

Fractional exretion of sodium and potassium were determined according to the following formula $\mathrm{FE}_{\mathrm{x}}=(\mathrm{uX}$ * $\mathrm{V} /$ pcreatinine/pX* ucreatinine)/GFR, where $\mathrm{uX}$ and $\mathrm{pX}$ are urine and plasma concentrations respectively, and $\mathrm{V}$ is the flow in $\mathrm{ml} / \mathrm{min}$ during GFR measurement.

\section{Urinary excretion of $A Q P 2$ and $E N a C_{Y}$}

Urine samples were kept frozen at $-20^{\circ} \mathrm{C}$ until assayed. U-AQP2 was measured by radioimmunoassay as previously described [20,21]. Antibodies were raised in rabbits to a synthetic peptide corresponding to the $15 \mathrm{COOH}$ terminal amino acids in human AQP2 to which was added an $\mathrm{NH}_{2}$-terminal cystein for conjugation and affinity purification. Minimal detection level was $34 \mathrm{pg} /$ tube/tube. The coefficients of variation were 11.7\% (inter-assay) and $5.9 \%$ (intra-assay). U-ENaCY was measured by radioimmunoassay as previously described [22]. Antibodies were raised against the synthetic $\mathrm{ENaC}_{\gamma}$ peptide in rabbits and affinity purified as described previously [23]. Minimal detection level was $48 \mathrm{pg} /$ tube. The coefficients of variation were 14\% (inter-assay) and 6.7\% (intra-assay). The anti-AQP2 antibody was a gift from Soeren Nielsen, The Water and Salt Research Center, Institute of Anatomy, Aarhus University, Denmark.

\section{Vasoactive hormones in plasma}

Blood samples for measurements of vasoactive hormones were centrifuged for 10 minutes at $2200 \mathrm{G}$ and $4{ }^{\circ} \mathrm{C}$. Plasma was separated from blood cells and kept frozen until assayed. PRC was determined using an immunoradiometric assay from CIS Bio International, Gif-Sur-Yvette Cedex, France. Minimal detection level was $1 \mathrm{pg} / \mathrm{ml}$. The coefficients of variation were $0.9-3.6 \%$ (intra-assay) and $3.7-5.0 \%$ (inter-assay) in the range of $4-263 \mathrm{pg} / \mathrm{ml}$. Aldo was determined by RIA using a kit from Demeditec Diagnostics $\mathrm{GmbH}$, Kiel, Germany. Minimal detection level was 25 $\mathrm{pmol} / \mathrm{l}$. The coefficients of variation were $9.0 \%$ (inter-assay) and 8.5\% (intra-assay). Ang II and AVP were extracted from plasma with $\mathrm{C}_{18}$ Sep-Pak (Water associates, Milford, MA, USA), and subsequently determined by radioimmunoassay $[24,25]$. The antibody against Ang II was obtained from the Department of Clinical Physiology, Glostrup Hospital, Denmark. Minimal detection level was $2 \mathrm{pmol} / \mathrm{L}$. The coefficients of variation were 12\% (inter-assay) and $8 \%$ (intraassay). The antibody against AVP was a gift from Professor Jacques Dürr, Miami, Fl, USA. Minimal detection level was $0.2 \mathrm{pmol} / \mathrm{L}$. The coefficients of variation were $13 \%$ (interassay) and $9 \%$ (intra-assay).

\section{Brachial and central blood pressure}

Bbrachial BP pressure was measured using an oscillometer (Omron 705IT) and recorded in accordance with recommendations from the European Society of Hypertension. Brachial systolic and diastolic blood pressure was recorded as the average of duplicate measures. Central BP was measured using applanation tonometry. Recordings of PWA and carotid-femoral PWV were obtained by applanation tonometry (SphygmoCor ${ }^{\circ}$ CPV system, AtCor Medical, Sydney, Australia) as double-recordings by a trained observer. Only duplicate recording meeting the quality requirements were included in the final analysis. An operator index of 80 or more was required to accept recordings of a peripheral pulse-wave form. The variation was measured in 10 healthy subjects. The participants rested for 15 minutes 
in a supine position followed by measurements of brachial blood pressure, PWA and PWV. For intra-observer variation, five measurements were performed by each observer in all ten subjects. For each subject, a coefficient of variation $(\mathrm{CV})$ was calculated for each observer. The mean values of CV's regarding the ten measurements for each observer were 4-6\% for PWV (Observer 1: 6.4\%, Observer 2: $3.8 \%$, and Observer 3: 5.6\%), and in the same range for PWA (Observer 1: 4.3\%, Observer 2: 5.0\%, and Observer 3: $5.7 \%)$. The inter-observer variation was calculated as means of $\mathrm{CV}$ of the average values obtained by the three observers for ten subjects (PWV: 7.5\%, PWA: 5.6\%).

\section{Routine analyses}

Sodium, albumin, hemoglobin and glucose were measured by routine methods in Department of Clinical Biochemistry, Holstebro Hospital.

\section{Statistics}

Statistical analyses were performed using IBM SPSS statistics version 20.0.0 (SPSS Inc., Chicago, IL, USA). General Linear Model Repeated Measures was used for comparison between and within subjects to test differences between placebo and tolvaptan treatment group at baseline, during and after infusion of L-NMMA. Post-hoc Bonferoni test was used to compare differences within groups at baseline and during L-NMMA infusion, and at baseline and after L-NMMA infusion.

Paired sample $t$-test was performed to compare differences between treatment groups at baseline, during and after infusion of L-NMMA. Statistical significance was at $<0.05$ in all analyses. Data with normal distribution are reported as means $\pm \mathrm{SD}$. Non-parametric test was performed for data with non-normal distribution, and are reported as medians \pm interquartile range or medians with $25^{\text {th }}$ and $75^{\text {th }}$ percentiles.

\section{Results}

\section{Demographics}

We allocated 29 subjects to the study. Ten of the participants were excluded due to lack of compliance (2), difficulties to gain intravenous access (3), arterial hypertension and/or medication (2), withdrawal of consent (3). Nineteen participants completed the study, 12 females and 7 males, with a median age of $25 \pm 4$ years, weight $72.3 \pm$ $13.4 \mathrm{~kg}$, body mass index $24 \pm 3.4 \mathrm{kgm}^{-2}$. p-sodium $141 \pm$ $1.7 \mathrm{mmol} / \mathrm{L}, \mathrm{p}$-potassium $3.8 \pm 0.3 \mathrm{mmol} / \mathrm{L}, \mathrm{p}$-creatinine $77.1 \pm 18.7 \mu \mathrm{mol} / \mathrm{L}$. Systolic brachial blood pressure (SBP) $122 \pm 9 \mathrm{mmHg}$, diastolic brachial blood pressure (DBP) $70 \pm 7 \mathrm{mmHg}$.

\section{Urine collection before the examination day}

Table 1 shows the values of the 24 hours urine collection before each examination day. There were no significant
Table 1 Urine output (UO), free water clearance $\left(\mathrm{C}_{\mathrm{H} 2 \mathrm{O}}\right)$, urinary excretion of AQP2 (u-AQP2), ENaC (u- ENaC $C_{\gamma}$ ), sodium ( $u-\mathrm{Na}$ ) and potassium (u-K) during a 24-hours urine collection before each examination day in a randomized, placebo-controlled, double-blind, crossover study of 19 healthy subjects

\begin{tabular}{|c|c|c|c|}
\hline & \multicolumn{2}{|c|}{ Before each examination day } & \multirow{2}{*}{$\underset{\text { (paired } t \text {-test) }}{\mathrm{p}}$} \\
\hline & Placebo & Tolvaptan & \\
\hline UO (ml/24 h) & $1969 \pm 448$ & $2025 \pm 514$ & 0.51 \\
\hline $\mathrm{C}_{\mathrm{H} 2 \mathrm{O}}(\mathrm{ml} / \mathrm{min})$ & $-0.46 \pm 0.61$ & $-0.47 \pm 0.57$ & 0.99 \\
\hline U-AQP2 (ng/min) & $1.10 \pm 0.32$ & $1.12 \pm 0.27$ & 0.57 \\
\hline U-ENaCY (pg/min) & $716 \pm 374$ & $705 \pm 376$ & 0.87 \\
\hline $\mathrm{U}-\mathrm{Na}(\mathrm{mmol} / 24 \mathrm{~h})$ & $103 \pm 11$ & $101 \pm 8$ & 0.652 \\
\hline U-K (mmol/24 h) & $55 \pm 19$ & $51 \pm 14$ & 0.472 \\
\hline
\end{tabular}

Data are shown as means with $\pm \mathrm{SD}$. Paired $t$-test was used for comparison between groups.

differences in $\mathrm{UO}, \mathrm{C}_{\mathrm{H} 2 \mathrm{O}}, \mathrm{u}-\mathrm{AQP} 2, \mathrm{u}-\mathrm{ENaC}_{\gamma}$ and $\mathrm{u}-\mathrm{Na}$ between the two treatment periods.

\section{GFR}

At baseline, GFR was the same after both treatments (Table 2). In response to L-NMMA infusion, GFR was significantly reduced during both treatments, but no differences were found between treatments. The relative decreases in GFR were similar after placebo and tolvap$\tan$ (Figure 1).

\section{Tubular handling of water and sodium}

Absolute values of $\mathrm{UO}$ and $\mathrm{C}_{\mathrm{H} 2 \mathrm{O}}$ after treatment with placebo and tolvaptan are shown in Table 2. The relative changes after L-NMMA infusions are shown in Figure 1. At baseline, $\mathrm{UO}$ and $\mathrm{C}_{\mathrm{H} 2 \mathrm{O}}$ were significantly higher during tolvaptan treatment compared with placebo $(\mathrm{p}=0.009$ and $\mathrm{p}=0.002$, respectively). During L-NMMA infusion, UO and $\mathrm{C}_{\mathrm{H} 2 \mathrm{O}}$ were significantly decreased after both treatments. However, $\mathrm{UO}$ and $\mathrm{C}_{\mathrm{H} 2 \mathrm{O}}$ were approximately $30 \%$ lower in the first 30 minutes (Period: 90-120 min) during L-NMMA infusion in the placebo group compared to the tolvaptan group ( $\mathrm{p}=0.026$ and 0.009 respectively). During the following 30 minutes (period: $120-150 \mathrm{~min}$.), $\mathrm{UO}$ and $\mathrm{C}_{\mathrm{H} 2 \mathrm{O}}$ were similar. The relative decrease in $\mathrm{C}_{\mathrm{H} 2 \mathrm{O}}$ and $\mathrm{UO}$ were significant within both treatments, but the relative decrease in UO and $\mathrm{C}_{\mathrm{H} 2 \mathrm{O}}$ were significantly more pronounced in the tolvaptan group ( $\mathrm{p}=0.018$ and $\mathrm{p}=0.008$ respectively).

At baseline, Table 2 shows that $\mathrm{FE}_{\mathrm{Na}}$ was similar during both treatments. During L-NMMA infusion, $\mathrm{FE}_{\mathrm{Na}}$ was significantly decreased and to the same extent during both treatments.

\section{$\mathrm{U}-\mathrm{AQP} 2$ and U-ENaC}

During baseline conditions, $\mathrm{u}-\mathrm{AQP} 2$ was the same after tolvaptan and placebo (Table 2). In response to L-NMMA 
Table 2 Effect of tolvaptan at baseline and during inhibition of the nitric oxide system on GFR (51-CrEDTA-clearance), urinary output $(\mathrm{OU})$, free water clearance $\left(\mathrm{C}_{\mathrm{H} 2 \mathrm{O}}\right)$, urinary aquaporin2 excretion rate (u-AQP2), urinary ENaC $\mathrm{C}_{\gamma}$ excretion rate $\left(\mathrm{u}-\mathrm{ENaC}_{\mathrm{\gamma}}\right)$ and fractional excretion of sodium $\left(\mathrm{FE}_{\mathrm{Na}}\right)$ in a randomized, double-blind, placebo-controlled, crossover study of 19 healthy subjects

\begin{tabular}{|c|c|c|c|c|c|c|}
\hline \multirow[t]{2}{*}{ Periods } & \multirow[t]{2}{*}{ Baseline } & \multicolumn{2}{|c|}{ L-NMMA } & \multicolumn{2}{|c|}{ Post infusion } & \multirow{2}{*}{$\stackrel{P}{P}$} \\
\hline & & $90-120 \mathrm{~min}$ & $120-150 \mathrm{~min}$ & $150-180 \mathrm{~min}$ & $180-210 \mathrm{~min}$ & \\
\hline \multicolumn{7}{|l|}{${ }^{51} \mathrm{Cr}$-EDTA-clearance $(\mathrm{ml} / \mathrm{min} / 1.73 \mathrm{~m} 2)$} \\
\hline Placebo & $99 \pm 5$ & $93 \pm 10^{* * *}$ & $92 \pm 8^{* * *}$ & $94 \pm 8^{* * *}$ & $97 \pm 9$ & \multirow{2}{*}{0.522} \\
\hline Tolvaptan & $97 \pm 9$ & $91 \pm 12^{* * *}$ & $93 \pm 9^{* * *}$ & $90 \pm 12^{* * *}$ & $94 \pm 12$ & \\
\hline \multicolumn{7}{|l|}{ p (GLM between) 0.527} \\
\hline $\mathrm{p}$ (paired t-test, between) & 0.403 & 0.533 & 0.583 & 0.118 & 0.197 & \\
\hline \multicolumn{7}{|l|}{ UO (ml/min) } \\
\hline Placebo & $7.4 \pm 1.3$ & $3.0 \pm 1.2^{* * *}$ & $4.2 \pm 1.3^{* * *}$ & $4.9 \pm 1.2^{* * *}$ & $6.3 \pm 1.1$ & \multirow{2}{*}{$<0.0001$} \\
\hline Tolvaptan & $8.9 \pm 1.7$ & $4.3 \pm 1.8^{* * *}$ & $4.2 \pm 1.8^{* * *}$ & $4.9 \pm 1.3^{* * *}$ & $5.7 \pm 1.6$ & \\
\hline \multicolumn{7}{|l|}{ p (GLM between) 0.230} \\
\hline $\mathrm{p}$ (paired t-test, between) & 0.009 & 0.026 & 0.965 & 0.861 & 0.124 & \\
\hline \multicolumn{7}{|l|}{$\mathrm{C}_{\mathrm{H} 2 \mathrm{O}}(\mathrm{ml} / \mathrm{min})$} \\
\hline Placebo & $4.6 \pm 1.1$ & $2.9 \pm 1.0^{* * *}$ & $3.9 \pm 1.2^{* * *}$ & $4.6 \pm 1.0^{* * *}$ & $5.9 \pm 1.0$ & \multirow{2}{*}{$<0.0001$} \\
\hline Tolvaptan & $5.8 \pm 1.1$ & $4.4 \pm 1.8^{*}$ & $4.0 \pm 1.6^{*}$ & $3.9 \pm 1.2$ & $5.2 \pm 1.4$ & \\
\hline \multicolumn{7}{|l|}{ p(GLM between) 0.185} \\
\hline $\mathrm{p}$ (paired $t$-test, between) & 0.002 & 0.009 & 0.756 & 0.063 & 0.097 & \\
\hline \multicolumn{7}{|l|}{ u-AQP2 (ng/min) } \\
\hline Placebo & $1.30 \pm 0.27$ & $1.04 \pm 0.36^{*}$ & $0.99 \pm 0.19^{* * *}$ & $1.06 \pm 0.24^{* * *}$ & $1.11 \pm 0.20$ & \multirow{2}{*}{0.444} \\
\hline Tolvaptan & $1.32 \pm 0.28$ & $1.08 \pm 0.39$ & $1.04 \pm 0.23^{* * *}$ & $1.17 \pm 0.32^{* * *}$ & $1.10 \pm 0.21$ & \\
\hline \multicolumn{7}{|l|}{ p (GLM between) 0.614} \\
\hline $\mathrm{p}$ (paired $t$-test, between) & 0.771 & 0.427 & 0.382 & 0.044 & 0.665 & \\
\hline \multicolumn{7}{|l|}{$\mathrm{u}-\mathrm{ENaC}_{\mathrm{\gamma}}(\mathrm{pg} / \mathrm{min})$} \\
\hline \multirow[t]{2}{*}{ Placebo } & 476 & & 367 & & $442^{* *}$ & \\
\hline & $(398 ; 640)$ & & $(296 ; 756)$ & & $(308 ; 605)$ & \\
\hline \multirow[t]{2}{*}{ Tolvaptan } & 320 & & $430 * *$ & & $567^{* * *}$ & \\
\hline & $(281 ; 379)$ & & $(361 ; 492)$ & & $(322 ; 537)$ & \\
\hline p (Wilcoxon's signed rank test, between) & $<0.001$ & & 0.355 & & 0.227 & \\
\hline \multicolumn{7}{|l|}{$\mathrm{FE}_{\mathrm{Na}}$} \\
\hline Placebo & $1.17 \pm 0.62$ & $0.77 \pm 0.35^{*}$ & $0.78 \pm 0.37^{*}$ & $1.12 \pm 0.41$ & $1.07 \pm 0.35$ & 0.945 \\
\hline Tolvaptan & $1.29 \pm 0.45$ & $0.89 \pm 0.34^{*}$ & $0.87 \pm 0.32^{*}$ & $1.22 \pm 0.30$ & $1.18 \pm 0.30$ & \\
\hline \multicolumn{7}{|l|}{ p (GLM between) 0.328} \\
\hline $\mathrm{p}$ (paired $t$-test, between) & 0.326 & 0.073 & 0.073 & 0.198 & 0.200 & \\
\hline
\end{tabular}

Data are shown as mean with \pm SD or medians with $25^{\text {th }}$ and $75^{\text {th }}$ percentiles in parentheses. General linear model (GLM) with repeated measures was performed for comparison within the group and intervention as between subject factor. Post-hoc Bonferoni test $\left(^{*}\right)$ was used for comparison of infusion period $90-150$ min vs baseline and post infusion period $150-210$ vs baseline. Paired $t$-test or Wilcoxon signed rank test was used for comparison between treatment group at baseline and during infusion period $90-150 \mathrm{~min}$, and at baseline and post infusion period $150-210 \mathrm{~min} .{ }^{*} \mathrm{p}<0.05 ;^{* *} \mathrm{p}<0.001 ;^{* * *} \mathrm{p}<0.0001$.

infusion, u-AQP2 decreased significantly and to the same extent after both treatments. The relative changes in $\mathrm{u}$ AQP2 were similar during and after L-NMMA infusion in the treatment groups (Figure 1).

$\mathrm{U}-\mathrm{ENaC}_{\gamma}$ was approximately $33 \%$ lower during tolvaptan treatment compared to placebo at baseline $(\mathrm{p}=0.002)$.
During L-NMMA infusion, $\mathrm{u}-\mathrm{ENaC}_{\gamma}$ was reduced by LNMMA infusion after placebo, whereas a significant increase was measured after tolvaptan $(\mathrm{p}<0.001)$. $\mathrm{U}-\mathrm{ENaC}_{\gamma}$ decreased $9.76 \pm 23.94 \%$ after placebo and increased $33.40 \pm 21.86 \%$ after tolvaptan in the L-NMMA infusion period (Figure 2). 


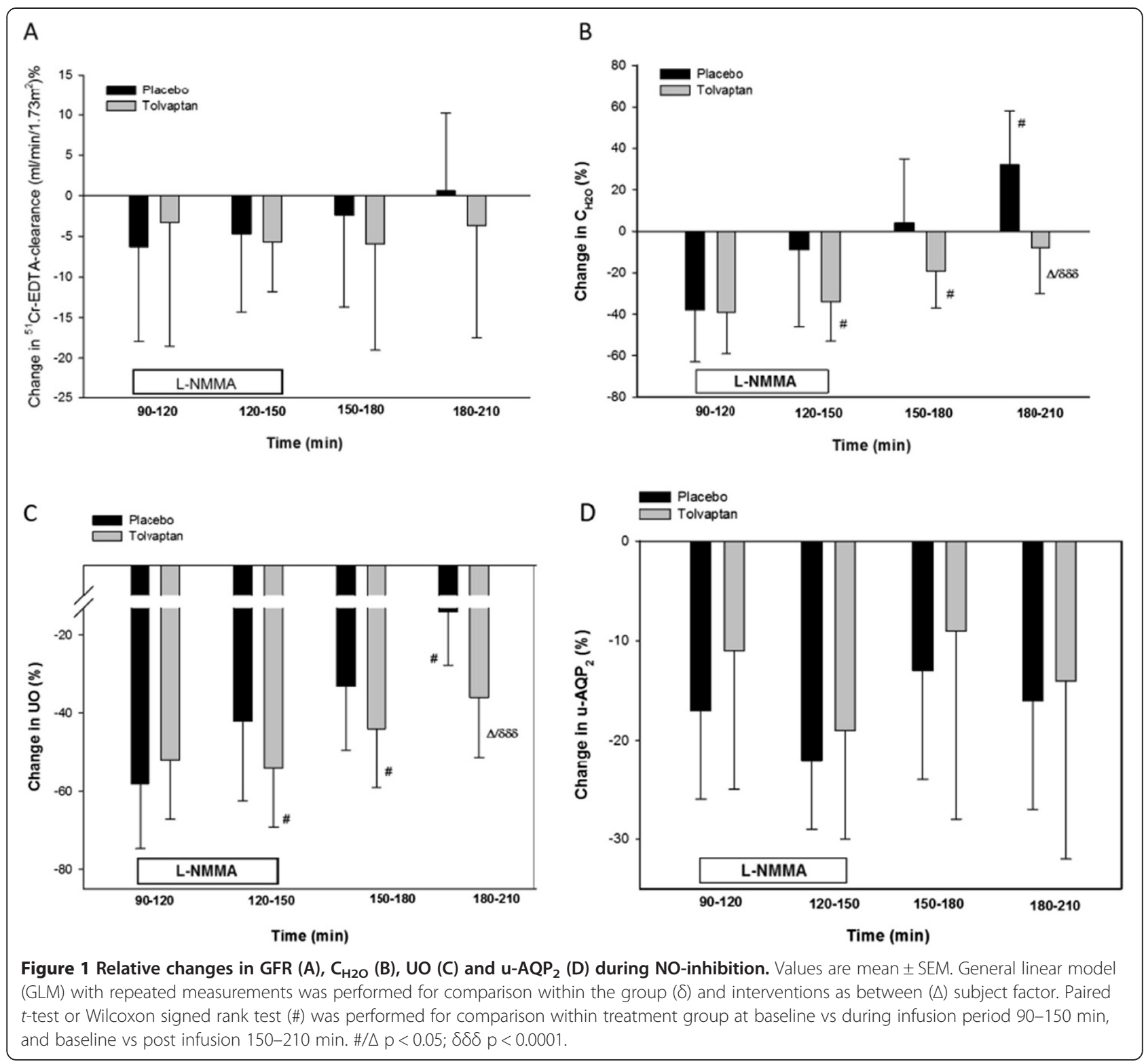

Plasma sodium, plasma osmolarity and plasma potassium During baseline conditions, $\mathrm{p}-\mathrm{Na}, \mathrm{p}-\mathrm{Osm}$ and $\mathrm{p}-\mathrm{K}$ were significantly higher in the tolvaptan group compared to the placebo group (Table 3). In response to L-NMMA infusion, $\mathrm{p}-\mathrm{Na}$ and $\mathrm{p}-\mathrm{Osm}$ remained significantly higher duing tolvaptan treatment compared with placebo, whereas no differences were measured in $\mathrm{p}-\mathrm{K}$ between the treatments.

\section{Vasoactive hormones}

At baseline, PRC, p-AngII and P-Aldo were the same after tolvaptan and placebo (Table 4). PRC was significantly decreased during inhibition of NO synthesis in both treatment groups $(\mathrm{p}<0.001)$, but no difference was observed between groups. P-AngII fell significantly only in the tolvaptan group $(\mathrm{p}=0.001)$, but tended to fall also during placebo. P-Aldo was unchanged during both treatments.

A highly significant and sustained 3-fold increase in pAVP was measured during treatment with tolvaptan compared to placebo (Placebo: $0.20 \pm 0.15$ vs. $0.70 \pm 0.45 \mathrm{pg} / \mathrm{ml}$, $\mathrm{p}<0.0001)$. P-AVP did not change from baseline levels during L-NMMA infusion.

\section{Effects of L-NMMA on brachial bloodpressure}

Within the first five minutes of L-NMMA bolus infusion, BP peaked in both groups and then gradually declined over the first $20 \mathrm{~min}$ of infusion (Figure 3). During the remaining 40 minutes of infusion, BP changes were similar in both groups $(\mathrm{p}=0.221$ for SBP and $\mathrm{p}=0.678$ for DBP with GLM). An average of the six measurements from the 


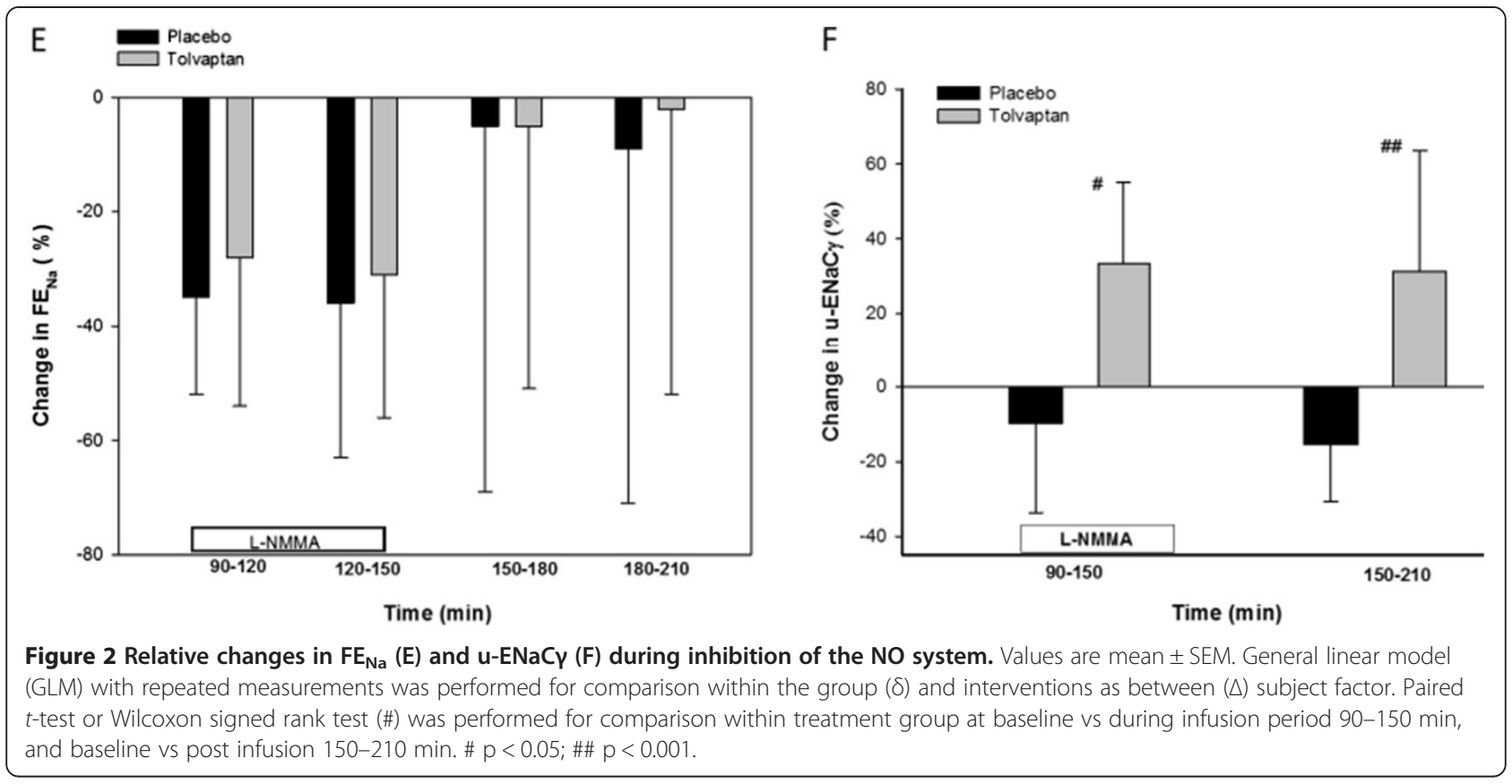

last 40 minutes of L-NMMA infusion was compared to baseline blood pressure. L-NMMA caused a significant increase in SBP $(4 \pm 3 \mathrm{mmHg}$ in placebo vs. $3 \pm$ $2 \mathrm{mmHg}$ in tolvaptan) and bDBP $(7 \pm 6 \mathrm{mmHg}$ in placebo vs. $6 \pm 7 \mathrm{mmHg}$ in tolvaptan). No significant differences were found between treatments $(\mathrm{p}=0.374$. for SBP and $\mathrm{p}=0.606$ for $\mathrm{DBP}$ ).
Effects of tolvaptan on brachial blood pressure

At baseline, SBP and DBP did not differ between treatments, whereas pulse rate was significantly higher in the placebo group (Table 5). L-NMMA infusion induced a significant increase in both SBP and DBP during both treatments, but differences were measured between treatments. During LNMMA infusion, pulse rate fell similarly in both groups.

Table 3 Effect of tolvaptan at baseline and during inhibition of the nitric oxide system on plasma concentration of sodium and potassium and plasma osmolality in a randomized, placebo-controlled, double-blind, crossover study of 19 healthy subjects

\begin{tabular}{|c|c|c|c|c|c|c|}
\hline \multirow{2}{*}{ Periods } & \multirow{2}{*}{$\begin{array}{l}\text { Baseline } \\
\text { 0-90 min }\end{array}$} & \multicolumn{2}{|c|}{ L-NMMA } & \multicolumn{2}{|c|}{ Post infusion } & \multirow{2}{*}{$\stackrel{p}{\text { (GLM-within) }}$} \\
\hline & & $90-120 \mathrm{~min}$ & $120-150 \mathrm{~min}$ & $150-180 \mathrm{~min}$ & $180-210 \mathrm{~min}$ & \\
\hline \multicolumn{7}{|l|}{ p-sodium (mmol/l) } \\
\hline Placebo & $140 \pm 2$ & $139 \pm 1$ & $139 \pm 2^{* *}$ & 139. \pm 2 & $138 \pm 2^{* *}$ & \multirow{3}{*}{0.272} \\
\hline Tolvaptan & $143 \pm 2$ & $143 \pm 2$ & $141 \pm 3^{* *}$ & $141 \pm 2$ & $141 \pm 2^{*}$ & \\
\hline P (Wilcoxon's signed rank test) & 0.001 & 0.001 & 0.002 & 0.001 & 0.001 & \\
\hline \multicolumn{7}{|l|}{ p- osm (mosm/kg) } \\
\hline Placebo & $285 \pm 3$ & $285 \pm 4$ & $283 \pm 4^{* *}$ & $284 \pm 4^{* *}$ & $283 \pm 4^{* * *}$ & \multirow{2}{*}{0.352} \\
\hline Tolvaptan & $291 \pm 3$ & $291 \pm 3$ & $291 \pm 3$ & $291 \pm 3$ & $290 \pm 3$ & \\
\hline \multicolumn{7}{|l|}{ p (GLM between) <0.0001 } \\
\hline $\mathrm{p}$ (paired t-test) & $<0.001$ & $<0.001$ & $<0.001$ & $<0.001$ & $<0.001$ & \\
\hline \multicolumn{7}{|l|}{ p-potassium (mmol/l) } \\
\hline Placebo & $3.8 \pm 0.2$ & $3.8 \pm 0.3$ & $3.9 \pm 0.3$ & $3.9 \pm 0.2$ & $3.8 \pm 0.1$ & \multirow{2}{*}{0.325} \\
\hline Tolvaptan & $3.9 \pm 0.2$ & $3.9 \pm 0.2$ & $4.0 \pm 0.2$ & $4.0 \pm 0.1$ & $4.0 \pm 0.1$ & \\
\hline p (Wilcoxon's signed rank test) & 0.028 & 0.117 & 0.272 & 0.929 & 0.430 & \\
\hline
\end{tabular}

Data are shown as mean with \pm SD or medians with \pm interquartile range. General linear model (GLM) with repeated measures was performed for comparison within the group and intervention as between subjects factor. Post-hoc Bonferoni test $\left(^{*}\right)$ was used for comparison of infusion period $90-150$ min vs baseline and post infusion period $150-210$ vs baseline. Paired $t$-test or Wilcoxon signed rank test was used for comparison between treatment group at baseline vs during infusion period 90-150 min, and at baseline vs post infusion period $150-210$ min. ${ }^{*} \mathrm{p}<0.05 ;{ }^{* *} \mathrm{p}<0.001{ }^{* * *} \mathrm{p}<0.0001$. 
Table 4 Effect of tolvaptan at baseline and during inhibition of the nitric oxide system on plasma concentrations of renin(PRC), angiotensin II (P-AngII), aldosterone (P-aldo) and vasopressin (P-AVP) in a randomized, placebo-controlled, double-blind, crossover study of 19 healthy subjects

\begin{tabular}{|c|c|c|c|c|}
\hline \multirow{2}{*}{ Periods } & Baseline & L-NMMA & Post infusion & \multirow{2}{*}{$\begin{array}{c}P \\
\text { (GLM-within }\end{array}$} \\
\hline & 11:00 AM & $12: 00 \mathrm{AM}$ & 1:00 PM & \\
\hline \multicolumn{5}{|l|}{$\mathrm{PRC}(\mathrm{pg} / \mathrm{ml})$} \\
\hline Placebo & $8.1 \pm 4.3$ & $6.1 \pm 3.2^{* *}$ & $5.9 \pm 3.2^{* *}$ & \multirow{2}{*}{0.670} \\
\hline Tolvaptan & $9.9 \pm 6.8$ & $7.5 \pm 4.6^{* *}$ & $7.5 \pm 5.4^{* *}$ & \\
\hline \multicolumn{5}{|l|}{ p (GLM between) 0.305} \\
\hline $\mathrm{p}$ (paired t-test, between) & 0.101 & 0.050 & 0.038 & \\
\hline \multicolumn{5}{|l|}{ P-Angll (pg/ml) } \\
\hline Placebo & $9.5 \pm 4$ & $8.6 \pm 3.7$ & $8.0 \pm 3.3^{*}$ & \multirow{2}{*}{0.156} \\
\hline Tolvaptan & $11.9 \pm 6.2$ & $9.4 \pm 4.6^{*}$ & $9.5 \pm 5.1^{*}$ & \\
\hline \multicolumn{5}{|l|}{ p (GLM between) 0.686} \\
\hline $\mathrm{p}$ (paired t-test, between) & 0.094 & 0.403 & 0.117 & \\
\hline \multicolumn{5}{|l|}{ P- Aldo (pmol/L) } \\
\hline Placebo & $70 \pm 2$ & $75 \pm 2$ & $64 \pm 2$ & \multirow{2}{*}{0.949} \\
\hline Tolvaptan & $71 \pm 2$ & $78 \pm 2$ & $66 \pm 1$ & \\
\hline \multicolumn{5}{|l|}{ p (GLM between) 0.899} \\
\hline $\mathrm{p}$ (paired t-test, between) & 0.962 & 0.785 & 0.840 & \\
\hline \multicolumn{5}{|l|}{ P- AVP (pg/ml) } \\
\hline Placebo & $0.20 \pm 0.15$ & $0.20 \pm 0.15$ & $0.20 \pm 0.20$ & \\
\hline Tolvaptan & $0.70 \pm 0.45$ & $0.70 \pm 0.55$ & $0.70 \pm 0.60$ & \\
\hline p (Wilcoxon's signed rank test, between) & $<0.001$ & $<0.001$ & $<0.001$ & \\
\hline
\end{tabular}

Data are shown as mean with \pm SD or medians with \pm interquartile range. General linear model (GLM) with repeated measures was performed for comparison within the group and intervention as between subject factor. Post-hoc Bonferoni test ${ }^{*}$ ) was used for comparison of infusion period $90-150$ min vs baseline and post infusion period 150-210 vs baseline. Paired $t$-test or Wilcoxon signed rank test was used for comparison between treatment group at baseline vs during infusion period 90-150 minu, and at baseline vs post infusion period $150-210$ min. ${ }^{*} \mathrm{p}<0.05 ;{ }^{* *} \mathrm{p}<0.001 ;{ }^{* * *} \mathrm{p}<0.0001$.

\section{Central blood pressure}

At baseline, no difference was observed in PWV, cBP and AI between tolvaptan and placebo (Table 6). During LNMMA infusion, PWV increased significantly in the tolvaptan group $(p=0.012)$, whereas it remained unchanged in the placebo group. Systolic cBP and AI increased in the same way in the tolvaptan group, but remained unchanged in the placebo group. The changes were similar between groups. During l-NMMA infusion diastolic cBP increased significantly after both treatments.

\section{Discussion}

In the present study, we examined the effect of short-term treatment with tolvaptan on renal tubular function, vasoactive hormones and central hemodynamics, during basal conditions and during inhibition of the NO-system with L-NMMA in healthy subjects. During baseline conditions, tolvaptan increased $\mathrm{UO}$ and $\mathrm{C}_{\mathrm{H} 2 \mathrm{O}}$. An expected decrease in $\mathrm{u}-\mathrm{AQP} 2$ was not measured and counteracted by a threefold increase in p-AVP. Tolvaptan did not change $\mathrm{FE}_{\mathrm{Na}}$ and decreased $\mathrm{u}-\mathrm{ENaC \gamma}$ as expected. During NOinhibition, $\mathrm{UO}$ and $\mathrm{C}_{\mathrm{H} 2 \mathrm{O}}$ decreased after both treatments, but the decrease after tolvaptan was significantly more pronounced than after placebo. In contrast, $\mathrm{FE}_{\mathrm{Na}}$ was decreased similarly after both tolvaptan and placebo during NO-inhibition. U-AQP2 decreased to the same extent after both treatments, whereas $\mathrm{u}-\mathrm{ENaC} Y$ decreased after tolvap$\tan$ and increased after placebo. The present study is the first randomized, double-blinded, placebo-controlled crossover trial to measure the effect of tolvaptan on renal tubular function, vasoactive hormones and central hemodynamics during inhibition of the NO-system with L-NMMA. The dose of L-NMMA was based on a previous dose-response study [9]. We demonstrated an infusion method in which a steady NOS inhibition was obtained, and in a dose dependent manner L-MNNA infusion increased blood pressure.

\section{Renal handling of water}

During baseline conditions, $\mathrm{UO}$ and $\mathrm{C}_{\mathrm{H} 2 \mathrm{O}}$ increased after tolvaptan as expected. However, after NO- inhibition, the decrease in $\mathrm{UO}$ and $\mathrm{C}_{\mathrm{H} 2 \mathrm{O}}$ was significantly more pronounced after tolvaptan than placebo, but p-AVP was unchanged. Thus, tolvaptan seemed to potentiate the reduction 


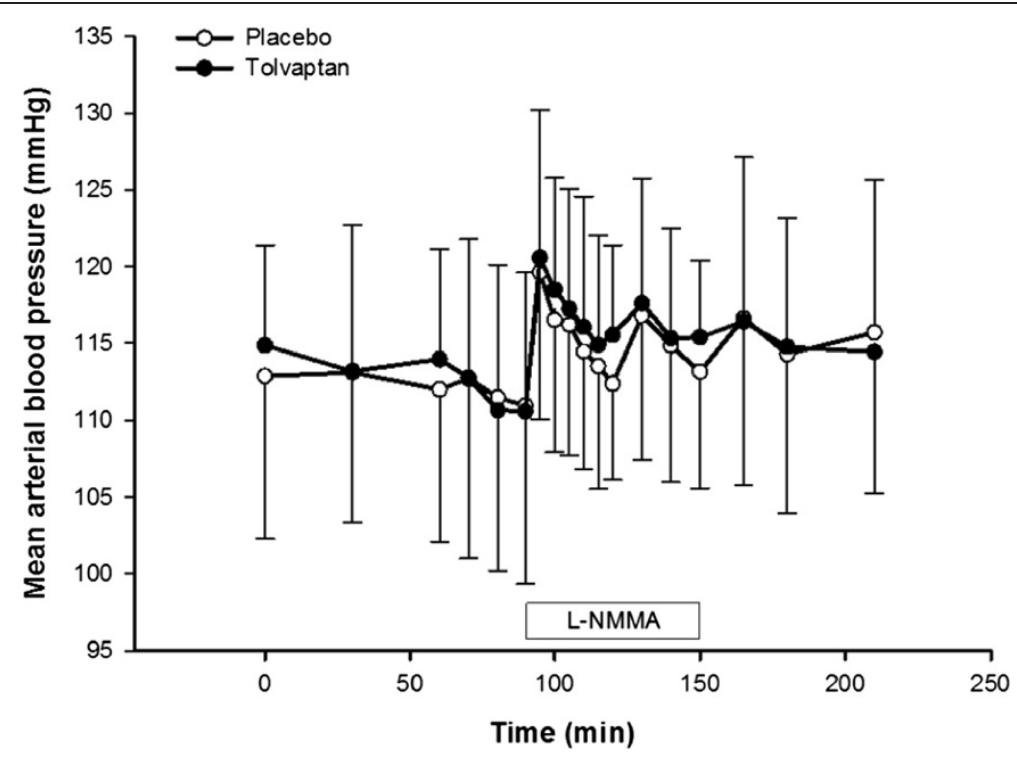

Figure 3 Effect of tolvaptan on bBP during inhibition of the NO system. Values are mean \pm SEM. Baseline brachial blood pressure (bBP) was defined as a mean of the four measurements 30 min prior to L-NMMA infusion. A stable bBP was achieved for the last 40 min of L-NMMA infusion. A mean of the six measurements from last 40 minutes of L-NMMA infusion was used to calculate changes from baseline. Post-hoc Bonferoni test was used for comparison of the mean of the last 40 min of infusion vs. baseline ( $p=0.011$ in placebo vs $p<0.0001$ in tolvaptan). Paired $t$-test was performed for comparison of the last 40 min of infusion vs. baseline between treatment groups.

in $\mathrm{UO}$ and $\mathrm{C}_{\mathrm{H} 2 \mathrm{O}}$ induced by L-NMMA. This could be explained by interference of $\mathrm{NO}$ with renal water excretion in the principal cells in the distal part of the nephron by a partly AVP-dependent mechanism, resulting in an increase in renal water excretion. This is a new and original observation. However, we cannot exclude an impact of $\mathrm{NO}$ on water excretion through an AVP-dependent mechanism, too.

In the distal part of the nephron, water is absorbed via AQP2 [1]. AVP exhibits a short-term effect through the cAMP pathway to regulate translocation of preformed AQP2 proteins from cytosolic vesicles to the apical plasma membrane of the collecting duct principal cells, thereby increasing water permeability across the collecting duct [3]. In addition, AVP exhibits a long-term effect through its action on the cAMP-responsive element in the AQP2 promoter site, thereby enhancing the AQP2 gene expression. This long-term regulation determines the abundance of AQP2 water channels available for the modulation of the apical membrane's water permeability $[1,26,27]$. This is in agreement with results in several experimental and clinical studies, which have demonstrated that treatment with vasopressin or 1-desamino-8-D-arginine-vasopressin (dDAVP), a V2R agonist, enhanced translocation of AQP2 water channels to the apical membrane and results in water retention [2]. Surprisingly, during baseline conditions, $\mathrm{u}-\mathrm{AQP} 2$ was the same after tolvaptan and placebo. However, the lack of decrease in U-AQP2 after tolvaptan can be attributed to the high level of p-AVP, which counteracts the effect of tolvaptan on the V2 receptor. Another explanation might be that the baseline period was too short to reflect a decrease in $\mathrm{u}-\mathrm{AQP} 2$ after tolvaptan. During NO inhibition, $\mathrm{u}-\mathrm{AQP} 2$ was suppressed to the same extent both after tolvaptan and placebo. Conflicting results have been reported regarding the effect of $\mathrm{NO}$ on AQP2 water channels. Some results suggested an effect via a cGMP dependent pathway, and others via an AVP dependent mechanism [28-30]. We demonstrated that $\mathrm{u}-\mathrm{AQP} 2$ fell in response to NO inhibition during both treatments. From the placebo treatment, our results strongly support the assumption that LNMMA decreased translocation of AQP2 channels to the apical membrane, and thereby reduced water absorption via reduced water transport via the AQP2 water channels. Tolvaptan treatment, however, potentiated the effect of LNMMA on $\mathrm{UO}$ and $\mathrm{C}_{\mathrm{H} 2 \mathrm{O}}$, and thereby increased the absorption of water significantly. This means that the increase in renal water excretion induced by $\mathrm{NO}$ seems to be at least partly mediated by an AVP-dependent mechanism. During NO-inhibition, the lack of differences in uAQP2 between tolvaptan and placebo can be attributed to the fact that $\mathrm{u}-\mathrm{AQP} 2$ was suppressed to a low level during both treatments by L-NMMA, and a difference between the two treatments could not be detected during this condition.

\section{Renal handling of sodium}

During baseline conditions, $\mathrm{FE}_{\mathrm{Na}}$ was the same after tolvaptan and placebo. L-NMMA reduced $\mathrm{FE}_{\mathrm{Na}}$ to the same extent after both treatments. The role of AVP in renal 
Table 5 Effect of tolvaptan at baseline and during inhibition of the nitric oxide system on brachial systolic- and diastolic blood pressure (SBP and DBP), and pulse rate in a randomized, placebo-controlled, double-blind, crossover study of 19 healthy subjects

\begin{tabular}{|c|c|c|c|c|c|c|}
\hline \multirow[t]{2}{*}{ Periods } & \multirow{2}{*}{$\begin{array}{l}\text { Baseline } \\
0-90 \text { min }\end{array}$} & \multicolumn{2}{|c|}{ L-NMMA } & \multicolumn{2}{|c|}{ Post infusion } & \multirow{2}{*}{$\underset{\text { (GLM-within) }}{\mathbf{p}}$} \\
\hline & & $90-120 \mathrm{~min}$ & $120-150 \mathrm{~min}$ & $150-180 \mathrm{~min}$ & $180-210 \mathrm{~min}$ & \\
\hline \multicolumn{7}{|l|}{ SBP (mmHG) } \\
\hline Placebo & $112 \pm 8$ & $113 \pm 9$ & $115 \pm 8^{*}$ & $115 \pm 9^{*}$ & $116 \pm 10^{*}$ & \multirow{2}{*}{0.170} \\
\hline Tolvaptan & $112 \pm 11$ & $115 \pm 9^{*}$ & $116 \pm 9^{*}$ & $116 \pm 10^{*}$ & $114 \pm 9$ & \\
\hline \multicolumn{7}{|l|}{ p (GLM between) 0.876} \\
\hline $\mathrm{p}$ (paired $t$-test, between) & 0.833 & 0.117 & 0.272 & 0.929 & 0.430 & \\
\hline \multicolumn{7}{|l|}{$\mathrm{DBP}(\mathrm{mmHg})$} \\
\hline Placebo & $61 \pm 5$ & $68 \pm 6^{* * *}$ & $67 \pm 6^{* * *}$ & $67 \pm 5^{*}$ & $65 \pm 7$ & \multirow{2}{*}{0.688} \\
\hline Tolvaptan & $63 \pm 5$ & $69 \pm 7^{* * *}$ & $68 \pm 6^{* * *}$ & $67 \pm 6$ & $66 \pm 7$ & \\
\hline \multicolumn{7}{|l|}{ p(GLM between) 0.606} \\
\hline $\mathrm{p}$ (paired t-test, between) & 0.049 & 0.467 & 0.259 & 0.907 & 0.454 & \\
\hline \multicolumn{7}{|l|}{ Pulse rate (BPM) } \\
\hline Placebo & $57 \pm 10$ & $52 \pm 9^{* * *}$ & $53 \pm 9^{* * *}$ & 55. $\pm 11^{* * *}$ & $58 \pm 12^{* * *}$ & \multirow{2}{*}{0.889} \\
\hline Tolvaptan & $55 \pm 10$ & $50 \pm 9^{* * *}$ & $51 \pm 9^{* * *}$ & $53 \pm 10^{* * *}$ & $56 \pm 11^{*}$ & \\
\hline \multicolumn{7}{|l|}{ p (GLM between) 0.527} \\
\hline $\mathrm{p}$ (paired $t$-test, between) & 0.016 & 0.022 & 0.007 & 0.013 & 0.003 & \\
\hline
\end{tabular}

sodium handling is debated. Activation of V2R by AVP increased water permeability of the luminal membrane through its action on AQP2, but also increased sodium absorption by of $\mathrm{ENaC}[2,5]$. According to studies in rats, the effect of AVP on ENaC depended on the plasma concentration of AVP [2]. Within the physiologic range, AVP promoted antinatriuresis in the distal nephron, mediated by V2R. In healthy subjects, Blanchard et al. [31] showed that the antinatriuretic effect of dDAVP was amiloride sensitive, and thus related to vasopressin's stimulatory effect on sodium absorption to be mediated by $\mathrm{ENaC}$. We found a lower $\mathrm{u}-\mathrm{ENaC} \gamma$ during tolvaptan treatment during baseline conditions, which is in agreement with a blockade of ENaC by VR2 antagonism. Our findings are in agreement with previous clinical studies of the effect of V2R agonism on sodium excretion by $\mathrm{ENaC}$ in patients with nephrogenic diabetes insipidus (NDI). Administration of dDAVP induced an antinatriuretic effect in NDI patients with an intact V2R, but not in those with defective V2R [32]. This is also in accordance with studies in rats, where a natriuretic effect was measured after V2R antagonism, but the aquaretic effect exceeded the natriuretic effect $[2,33]$. During NO-inhibition, u-ENaC $\gamma$ decreased after placebo and increased after tolvaptan. Thus, NO-inhibition antagonized the effect of tolvaptan on $\mathrm{ENaC}$. Although the variation in $\mathrm{u}-\mathrm{ENaC}_{\gamma}$ was considerable, this suggests that $\mathrm{NO}$ interferes with the transport via $\mathrm{ENaC}$ with an AVP- dependent mechanism, supporting the assumption that the AVP-cAMP pathway of ENaC regulation is relevant for sodium homeostasis in humans [28,32]. However, the decrease in sodium reabsorption after L-NMMA was not mediated by a reduced transport via $\mathrm{ENaC}$, but must be due to L-NMMA induced change in tubular sodium absorption at another location in the nephron.

\section{Vasoactive hormones}

We found no differences in PRC, p-Aldo and p-AngII at baseline in the two treatments, whereas p-AVP increased approximately 3 -fold in response to treatment with tolvap$\tan$. Most likely, this rise in endogenous p-AVP is a compensatory release of the hormone in response to the rise in p-osmolality induced by V2R antagonism [34]. During L-NMMA infusion, PRC fell after placebo and tolvaptan treatment, whereas p-ANG.II tended to fall after both treatment, but only significantly in the tolvaptan group. This is in good agreement with previous results from our group [9]. P-AVP remained unchanged during L-NMMA infusion in both treatment groups. Thus, NO does not seem to have a regulatory effect on the release of AVP.

\section{Central and brachial blood pressure}

We observed no changes in $\mathrm{bBP}$ and $\mathrm{cBP}$ during treatment with tolvaptan despite of the increase in p-AVP in the tolvaptan group. The effect of AVP on BP is mediated 


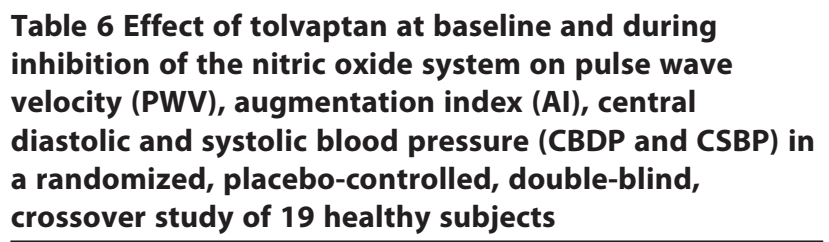

\begin{tabular}{lcc}
\hline Periods & Baseline & L-NMMA \\
\hline PWV(m/s) & $5.3 \pm 0.7$ & $5.5 \pm 0.5$ \\
Placebo & $5.3 \pm 0.6$ & $5.6 \pm 0.7^{*}$ \\
Tolvaptan & 0.652 & 0.929 \\
p (paired t-test, between) & & \\
Al & $0.0 \pm 18.7$ & $3.7 \pm 18.7$ \\
Placebo & $1.2 \pm 18.3$ & $7.2 \pm 17.5^{*}$ \\
Tolvaptan & 0.646 & 0.064 \\
p (paired t-test, between) & & \\
CSBP & $100 \pm 7$ & $105 \pm 4$ \\
Placebo & $100 \pm 4$ & $107 \pm 7^{*}$ \\
Tolvaptan & 0.871 & 0.351 \\
p (paired t-test, between) & & \\
CDBP & $66 \pm 12$ & $70 \pm 8^{*}$ \\
Placebo & $64 \pm 5$ & $68 \pm 8^{*}$ \\
Tolvaptan & 0.440 & 0.622 \\
p (Wilcoxon signed rank test, between) &
\end{tabular}

Data are shown as mean with \pm SD or medians with \pm interquartile range. Paired $t$-test or Wilcoxon signed rank test was used for comparison within (*) treatment group at baseline vs during infusion period 90-150 min, and at baseline vs post infusion period $150-210 \mathrm{~min} .{ }^{*} \mathrm{p}<0.05$

by its action on V1a receptors. Activation of V1a receptor increases BP by a direct effect on vascular smooth muscle cells, by which AVP stimulates vasoconstriction and increases BP [2]. It is well documented that treatment with V2 receptor antagonists causes compensatory increase in the plasma concentration of vasopressin, which stimulates the V1a receptors in the vascular smooth muscle cells and thus affect central and peripheral hemodynamics [2]. Our study is the first to measure both bBP and cBP during V2R antagonism before, during and after inhibition of systemic NO synthesis in healthy subjects. Applanation tonometry was performed under standardized conditions. The method is described and evaluated elsewhere [35]. We showed that a 3-fold increase in AVP was not sufficient to increase $\mathrm{BP}$ via an activation of $\mathrm{V} 1 \mathrm{aR}$, which is consistent with previous studies [2]. However, pulse rate values were approximately 2 beats per minute lower in the tolvaptan group compared to the placebo group. Most likely, this slight decrease in pulse rate is due to increased baroreceptor sensitivity exerted by AVP [36,37]. However this had no net effect on BP. L-NMMA infusion caused an increase in bBP and $\mathrm{cBP}$ to the same extent during both treatments. The results are in agreement with the results recently reported by our group [9] on the effect on systemic NO inhibition on BP. Thus, in the given dose of tolvaptan, no effect is measured on bBP and $\mathrm{cBP}$.

\section{Plasma levels of sodium, potassium and osmolality}

We found significantly higher $\mathrm{p}-\mathrm{Na}, \mathrm{p}-\mathrm{K}$ and p-osmolality in baseline during treatment with tolvaptan. The response is in agreement with previous clinical studies [12,17,33]. The SALT [17] investigators have demonstrated that serum sodium concentration was significantly higher within 8 hours after administration of tolvaptan $15 \mathrm{mg}$ compared to placebo. It should be noticed that their study was conducted in patients with hypervolemic and euvolemic hyponatremia. In response to $\mathrm{NO}$ inhibition, $\mathrm{p}$-osm and $\mathrm{p}-\mathrm{K}$ did not change. In contrast, $\mathrm{p}-\mathrm{Na}$ decreased in both treatment groups in the last 30 minutes of L-NMMA infusion. It is easily explained by the decrease of $\mathrm{UO}, \mathrm{C}_{\mathrm{H} 2 \mathrm{O}}$ and $\mathrm{u}-\mathrm{Na}$. Recently, our group reported similar results during L-NMMA infusion in healthy subjects [9].

\section{Strengths and limitations}

The major strength of the present study was the design as a randomized, double-blinded, placebo-controlled crossover trial in healthy subjects. Diet, sodium and fluid intake were predefined and controlled during the study to avoid confounding of the results. It is a weakness of the study that we did not measure total plasma or urine nitrite and nitrate as indices of $\mathrm{NO}$ synthesis to ensure abrogated systemic NO production. However, during L-NMMA infusion we measured an increase in mean arterial blood pressure and a decrease in GFR, UO, $\mathrm{C}_{\mathrm{H} 2 \mathrm{O}}, \mathrm{u}-\mathrm{AQP} 2$ and $\mathrm{FE}_{\mathrm{Na}}$ which clearly indicated NO inhibition.

\section{Conclusion}

During baseline conditions, tolvaptan increased renal water excretion. During NO-inhibition, the more pronounced reduction in renal water excretion after tolvaptan than after placebo indicates that NO promotes renal water excretion in the principal cells by at least a partly an AVPdependent mechanism. The lack of decrease in $\mathrm{u}$-AQP2 by tolvaptan could be explained by a counteracting effect of a three-fold increase in plasma vasopressin. The antagonizing effect of $\mathrm{NO}$-inhibition on $\mathrm{u}-\mathrm{ENaC}$ suggests that $\mathrm{NO}$ interferes with the transport via $\mathrm{ENaC}$ by an AVPdependent mechanism.

\section{Competing interests}

The authors declare that they have no competing interests.

\section{Authors' contributions}

SAT participated in the coordination of the study, recruitment of subjects, carried out the experiments, performed statistical analysis, interpretation of data and drafted the manuscript. FHM assisted in the experimental part and helped to draft the manuscript. JMJ assisted in the experimental part and helped to draft the manuscript. JNB helped to draft the manuscript. EBP conceived of the study, participated in the design of the study, was responsible for the laboratory analyses and helped to draft the article. All authors read and approved the final manuscript. 


\section{Acknowledgements}

We thank laboratory technicians Anne Mette Ravn, Kirsten Nygaard, Henriette Vorup Simonsen and Susan Milton Rasmussen for skillful technical assistance and commitment.

The study was supported by grants from Region Midt's Research Foundation for Health Science.

Received: 22 January 2014 Accepted: 19 June 2014

Published: 25 June 2014

\section{References}

1. Nielsen S, Kwon TH, Christensen BM, Promeneur D, Frokiaer J, Marples D: Physiology and pathophysiology of renal aquaporins. J Am Soc Nephrol 1999, 10(3):647-663.

2. Perucca J, Bichet DG, Bardoux P, Bouby N, Bankir L: Sodium excretion in response to vasopressin and selective vasopressin receptor antagonists. J Am Soc Nephrol 2008, 19(9):1721-1731.

3. Verbalis JG, Goldsmith SR, Greenberg A, Schrier RW, Sterns RH: Hyponatremia treatment guidelines 2007: expert panel recommendations. Am J Med 2007, 120(11 Suppl 1):S1-21.

4. Lauridsen TG, Vase H, Starklint J, Graffe CC, Bech JN, Nielsen S, Pedersen EB: Increased renal sodium absorption by inhibition of prostaglandin synthesis during fasting in healthy man. A possible role of the epithelial sodium channels. BMC Nephrol 2010, 11:28.

5. Loffing J, Korbmacher C: Regulated sodium transport in the renal connecting tubule (CNT) via the epithelial sodium channel (ENaC). Pflugers Arch 2009, 458(1):111-135.

6. Forstermann U, Sessa WC: Nitric oxide synthases: Regulation and function. Eur Heart J 2012, 33(7):829-37. 837a-837d.

7. Kurtz $\mathrm{A}$, Wagner $\mathrm{C}$ : Role of nitric oxide in the control of renin secretion. Am J Physiol 1998, 275(6 Pt 2):F849-F862.

8. Ortiz PA, Garvin JL: Role of nitric oxide in the regulation of nephron transport. Am J Physiol Renal Physiol 2002, 282(5):F777-F784.

9. Larsen T, Mose FH, Bech JN, Pedersen EB: Effect of nitric oxide inhibition on blood pressure and renal sodium handling: a dose-response study in healthy man. Clin Exp Hypertens 2012, 34(8):567-574.

10. Ali F, Guglin M, Vaitkevicius P, Ghali JK: Therapeutic potential of vasopressin receptor antagonists. Drugs 2007, 67(6):847-858.

11. Berl T, Quittnat-Pelletier F, Verbalis JG, Schrier RW, Bichet DG, Ouyang J, Czerwiec FS: Oral tolvaptan is safe and effective in chronic hyponatremia. J Am Soc Nephrol 2010, 21(4):705-712.

12. Elhassan EA, Schrier RW: Hyponatremia: diagnosis, complications, and management including V2 receptor antagonists. Curr Opin Nephrol Hypertens 2011, 20(2):161-168.

13. Ellison DH, Berl T: Clinical practice. The syndrome of inappropriate antidiuresis. N Engl J Med 2007, 356(20):2064-2072.

14. Kwon TH, Nielsen J, Knepper MA, Frokiaer J, Nielsen S: Angiotensin II AT1 receptor blockade decreases vasopressin-induced water reabsorption and AQP2 levels in NaCl-restricted rats. Am J Physiol Renal Physiol 2005, 288(4):F673-F684.

15. Lee YJ, Song IK, Jang KJ, Nielsen J, Frøkiaer J, Nielsen S, Kwon TH: Increased AQP2 targeting in primary cultured IMCD cells in response to angiotensin II through AT1 receptor. Am J Physiol Renal Physiol 2007, 292(1):F340-F350.

16. Miyazaki T, Fujiki H, Yamamura Y, Nakamura S, Mori T: Tolvaptan, an orally active vasopressin $\mathrm{V}(2)$-receptor antagonist - pharmacology and clinical trials. Cardiovasc Drug 2007, 25(1):1-13.

17. Schrier RW, Gross P, Gheorghiade M, Berl T, Verbalis JG, Czerwiec FS, Orlandi C: Tolvaptan, a selective oral vasopressin V2-receptor antagonist, for hyponatremia. N Eng/ J Med 2006, 355(20):2099-2112.

18. de Seigneux S, Nielsen J, Olesen ET, Dimke H, Kwon TH, Frøkiaer J, Nielsen S: Long-term aldosterone treatment induces decreased apical but increased basolateral expression of AQP2 in CCD of rat kidney. Am J Physiol Renal Physiol 2007, 293(1):F87-F99.

19. Wong NL, Tsui JK: Angiotensin II upregulates the expression of vasopressin V2 mRNA in the inner medullary collecting duct of the rat. Metabolism 2003, 52(3):290-295.

20. Graffe CC, Bech JN, Pedersen EB: Effect of high and low sodium intake on urinary aquaporin-2 excretion in healthy humans. Am J Physiol Renal Physiol 2012, 302(2):F264-F275.
21. Pedersen RS, Bentzen $H$, Bech JN, Pedersen EB: Effect of water deprivation and hypertonic saline infusion on urinary AQP2 excretion in healthy humans. Am J Physiol Renal Physiol 2001, 280(5):F860-F867.

22. Matthesen SK, Larsen T, Lauridsen TG, Vase H, Gjørup PH, Nykjær KM, Nielsen S, Pedersen EB: Effect of amiloride and spironolactone on renal tubular function, ambulatory blood pressure, and pulse wave velocity in healthy participants in a double-blinded, randomized, placebo-controlled, crossover trial. Clin Exp Hypertens 2012, 34(8):588-600.

23. Hager H, Kwon TH, Vinnikova AK, Masilamani S, Brooks HL, Frøkiaer J, Knepper MA, Nielsen S: Immunocytochemical and immunoelectron microscopic localization of alpha-, beta-, and gamma-ENaC in rat kidney. Am J Physiol Renal Physiol 2001, 280(6):F1093-F1106.

24. Pedersen EB, Danielsen H, Spencer ES: Effect of indapamide on renal plasma flow, glomerular filtration rate and arginine vasopressin in plasma in essential hypertension. Eur J Clin Pharmacol 1984, 26(5):543-547.

25. Pedersen EB, Eiskjaer H, Madsen B, Danielsen H, Egeblad M, Nielsen CB Effect of captopril on renal extraction of renin, angiotensin II, atrial natriuretic peptide and vasopressin, and renal vein renin ratio in patients with arterial hypertension and unilateral renal artery disease. Nephrol Dial Transplant 1993, 8(10):1064-1070.

26. Lauridsen TG, Vase H, Starklint J, Bech JN, Pedersen EB: Protein-enriched diet increases water absorption via the aquaporin-2 water channels in healthy humans. Nephrol Dial Transplant 2010, 25(8):2502-2510.

27. Lee J, Yoo K, Kim SW, Jung KH, Ma SK, Lee YK, Kim WY, Kim J, Choi KC Decreased expression of aquaporin water channels in denervated rat kidney. Nephron Physiol 2006, 103(4):170-178.

28. Baylis C: Nitric oxide deficiency in chronic kidney disease. Am J Physiol Renal Physiol 2008, 294(1):F1-F9.

29. Bouley R, Breton S, Sun T, McLaughlin M, Nsumu NN, Lin HY, Ausiello DA, Brown D: Nitric oxide and atrial natriuretic factor stimulate cGMP-dependent membrane insertion of aquaporin 2 in renal epithelial cells. J Clin Invest 2000, 106(9):1115-1126.

30. Klokkers J, Langehanenberg P, Kemper B: Atrial natriuretic peptide and nitric oxide signaling antagonizes vasopressin-mediated water permeability in inner medullary collecting duct cells. Am J Physiol Renal Physiol 2009, 297(3):F693-F703.

31. Blanchard A, Frank M, Wuerzner G, Peyrard S, Bankir L, Jeunemaitre X, Azizi M: Antinatriuretic effect of vasopressin in humans is amiloride sensitive, thus ENaC dependent. Clin J Am Soc Nephrol 2011, 6(4):753-759.

32. Bankir L, Fernandes S, Bardoux P, Bouby N, Bichet DG: Vasopressin-V2 receptor stimulation reduces sodium excretion in healthy humans. J Am Soc Nephrol 2005, 16(7):1920-1928.

33. Gheorghiade M, Gottlieb SS, Udelson JE, Konstam MA, Czerwiec F, Ouyang J, Orlandi C: Vasopressin v(2) receptor blockade with tolvaptan versus fluid restriction in the treatment of hyponatremia. Am J Cardiol 2006, 97(7):1064-1067.

34. Robertson GL: Vaptans for the treatment of hyponatremia. Nat Rev Endocrinol 2011, 7(3):151-161.

35. Matthesen SK, Larsen T, Vase H, Lauridsen TG, Pedersen EB: Effect of potassium supplementation on renal tubular function, ambulatory blood pressure and pulse wave velocity in healthy humans. Scand J Clin Lab Invest 2012, 72(1):78-86.

36. Berecek KH, Swords BH: Central role for vasopressin in cardiovascular regulation and the pathogenesis of hypertension. Hypertension 1990, 16(3):213-224

37. Luk J, Ajaelo I, Wong V, Wong J, Chang D, Chou L, Reid IA: Role of V1 receptors in the action of vasopressin on the baroreflex control of heart rate. Am J Physiol 1993, 265(3 Pt 2):R524-9.

doi:10.1186/1471-2369-15-100

Cite this article as: Al Therwani et al.: Effect of vasopressin antagonism on renal handling of sodium and water and central and brachial blood pressure during inhibition of the nitric oxide system in healthy subjects. BMC Nephrology 2014 15:100 